\title{
Erratum to: Stem Cell-Derived RPE Transplantation:The Feasibility and Advantages of Delivery as Monolayers
}

Odysseas Georgiadis, Lyndon da Cruz, and Peter Coffey

\section{Erratum to:}

Chapter 2 in: S.D. Schwartz et al. (eds.), Cellular Therapies for Retinal Disease,

DOI 10.1007/978-3-319-49479-1_2

The original version of this chapter contained a mistake. Co-author names were omitted in the originally published Chapter 2. Chapter 2 was written by Odysseas Georgiadis and Lyndon da Cruz with oversight and supervision of Peter Coffey. The author names and corresponding affiliations have been updated with this erratum.

The updated online version of this chapter can be found at https://dx.doi.org/10.1007/978-3-319-49479-1_2 